\title{
1 Predatory behaviour as a personality trait in a wild fish population
}

2 Andrew W. Szopa-Comley ${ }^{1}$, Callum Duffield ${ }^{2}$, Indar W. Ramnarine ${ }^{3}$, Christos C. Ioannou ${ }^{1}$

3 Author affiliations:

$4 \quad{ }^{1}$ School of Biological Sciences, University of Bristol, Bristol, BS8 1TQ, U.K.

$5 \quad{ }^{2}$ Institute of Integrative Biology, University of Liverpool, Leahurst Campus, Neston, CH64 7TE,

6 U.K.

$7 \quad{ }^{3}$ Department of Life Sciences, University of the West Indies at St. Augustine, St. Augustine,

8 Trinidad and Tobago.

9 Corresponding author (*): Andrew Szopa-Comley; address: School of Biological Sciences, Life

10 Sciences Building, 24 Tyndall Avenue, University of Bristol, Bristol, BS8 1TQ, U.K.; email:

11 a.szopa-comley@bristol.ac.uk

12 
$\underline{\text { Abstract }}$

21 Consistent inter-individual differences in behaviour (i.e. animal personality variation) can

22 influence a range of ecological and evolutionary processes, including predation. Variation

23 between individual predators in commonly measured personality traits, such as boldness and

24 activity, has previously been linked to encounter rates with their prey. Given the strong selection

25 on predators to respond to prey, individual predators may also vary consistently in their response

26 to prey in a manner that is specific to the context of predation. By studying wild piscivorous fish

27 (pike cichlids, Crenicichla frenata) in their natural environment using experimental presentations

28 of prey and control stimuli, we show that individual predators differ consistently in the amount

29 of time spent near prey. Crucially, these differences were not explained by the behaviour of the

30 same individuals in control presentations (the same apparatus lacking prey), suggesting that

31 variation in the response to prey reflects a 'predator personality trait' which is independent from

32 other individual traits (body size, boldness and/or neophobia) and environmental factors. Pike

33 cichlids which spent more time near prey also attacked prey at a higher rate. These findings

34 imply that the risk posed by individual predators cannot always be adequately predicted from

35 typically studied axes of personality variation.

$37 \underline{\text { Keywords }}$

38 animal personality, behavioural type, guppy, pike cichlid, predator-prey interactions, predation

39 risk 
41 Through their direct effect on prey abundance (Paine 1966) and non-lethal impact on prey

42 physiology and behaviour (Beckerman et al. 1997; Lima 1998), predators exert a strong

43 influence on the structure and composition of ecological communities (Schmitz et al. 2004). An

44 extensive body of research has explored how prey adjust their behaviour in response to changes

45 in predation risk (Lima and Dill 1990), and has revealed how the mere presence of nearby

46 predators can shape prey population dynamics and the abundance of resources at lower trophic

47 levels (Preisser et al. 2005; Peckarsky et al. 2008; Suraci et al. 2016). Predators, by contrast, are

48 often viewed as being behaviourally unresponsive, posing a fixed and uniform level of risk to

49 prey (Lima 2002). Increasingly this simplifying assumption is at odds with the evidence for

50 widespread consistent inter-individual differences in behaviour (also known as personality

51 variation) within natural populations (Bell et al. 2009). As the effects of variation within species

52 on ecological processes can often equal or outweigh the impact of differences between species

53 (Des Roches et al. 2018), determining how individual predators differ in their behaviour is key to

54 understanding their wider effects (Ioannou et al. 2008; Okuyama, 2008; Start and Gilbert 2017;

55 Michalko and Řežucha, 2018; Rhoades et al. 2019).

56 Empirical research has shown that inter-individual behavioural differences can influence

57 numerous ecological and evolutionary processes (Dall et al. 2012; Sih et al. 2012), ranging from

58 dispersal (Cote et al. 2010) to pair bonding (Firth et al. 2018). However, most studies have

59 focused on a limited number of traits, particularly boldness, exploration, activity, aggressiveness

60 and sociability (Réale et al. 2007), which are not necessarily the most ecologically relevant axes

61 of variation (Koski 2014). Although a wide variety of other behaviours are known to be

62 individually repeatable (reviewed in Bell et al. 2009), there have been few tests examining 
63 whether inter-individual variation in these behavioural traits is separate from, or correlated with,

64 frequently measured axes of variation. If commonly studied personality traits are not strongly

65 correlated with other repeatable behaviours that have greater ecological or evolutionary

66 relevance, this has implications for both how personality traits affect ecological and evolutionary

67 processes, as well as the selection imposed on different personality traits. Answering this

68 question may be particularly important for widespread ecological processes like predation, which is almost ubiquitous in animals across a diverse range of taxa and habitats.

70 The majority of studies exploring the consequences of personality differences for predator-prey

71 interactions have concentrated on variation in activity levels or boldness (Bell and Sih 2007),

72 behaviours which reflect the degree to which individuals prioritise gaining resources over risk

73 avoidance (Smith and Blumstein 2008). Bolder or more active prey are often more susceptible to

74 predation (Ballew et al. 2017; Hulthén et al. 2017), although this does not necessarily result in

75 positive correlations between boldness and survival (Moiron et al. 2020). The link between these

76 traits and individual movement patterns also suggests that bolder or more active predators are

77 also more likely to encounter prey (Spiegel et al. 2015). Consistent with the expected effect of

78 boldness on encounter rates, the interacting effects of prey and predator behavioural types have

79 been shown to determine prey survival (Pruitt et al. 2012; Chang et al., 2017). In seabird

80 populations, boldness also predicts inter-individual variation in how individual predators search

81 for prey (Patrick et al. 2017). As well as affecting encounter rates with prey, personality variation

82 influences the rate of prey consumption (Toscano and Griffen 2014), and individual predators

83 have also been shown to differ consistently in the time taken to detect, respond to or capture prey

84 (McGhee et al. 2013; Szopa-Comley et al., 2020; MacGregor et al., 2020). 
85 Quantifying behavioural variation in the wild avoids artefacts which can arise when behaviour is

86 expressed in laboratory conditions (Niemelä and Dingemanse 2014). Predator personality

87 variation will also be most relevant to the risk prey experience when prey are repeatedly exposed

88 to the same individual predators. This context arises in populations of the Trinidadian guppy

89 (Poecilia reticulata) in their natural habitats, where guppies can be confined to the same natural

90 river pools during the dry season as pike cichlids (Crenicichla frenata), their main predator

91 (Magurran 2005; Botham et al. 2006). Rather than engaging in lengthy pursuits, pike cichlids

92 typically track their prey visually before attacking in a rapid burst once they have approached

93 within close proximity (Walker et al., 2005; Heathcote et al., 2020). In this study, we

94 investigated inter-individual variation in predator behaviour by repeatedly presenting stimulus

95 shoals of guppies in situ (the prey treatment) over multiple days across a series of 16 discrete

96 natural river pools, and recorded the time spent near the stimulus by individually-identified pike

97 cichlids (Fig. 1). Similar methods of quantifying predation risk have also been shown to correlate

98 with anti-predator behaviour in this system (Croft et al. 2006). Importantly, we repeatedly

99 presented the same apparatus without prey as a control treatment in each pool. The response of

100 individual predators to the control should reflect variation in the individual traits or

101 environmental factors which are not specific to prey, including personality variation in boldness

102 and neophobia (during the initial presentation, the (empty) stimulus was entirely novel). For

103 example, whether or not individual pike cichlids were recorded approaching the control stimulus

104 should indicate their response to novel features within their environment. By comparing the

105 predator's behaviour in the prey and control treatments, we were therefore able to isolate inter-

106 individual variation in predatory behaviour (i.e. the response to prey) from factors that are not

107 specific to prey. 
a)

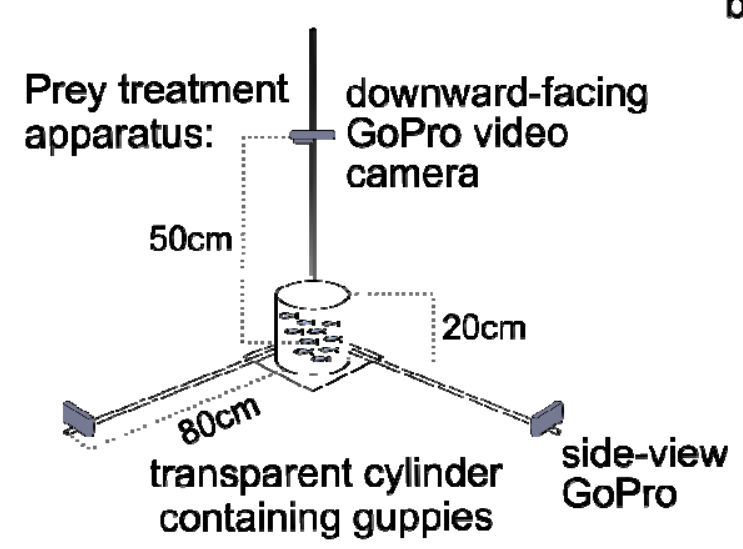

c)
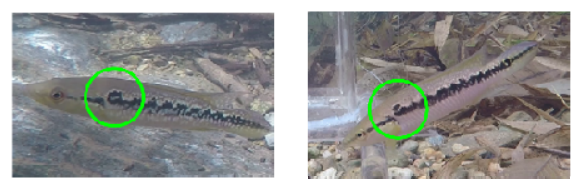

b)

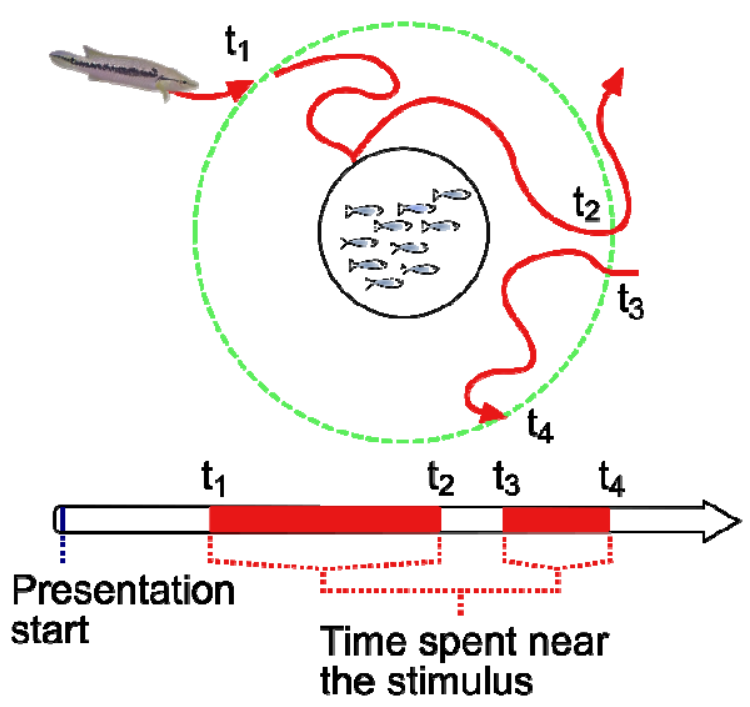

Time spent near the stimulus

Figure 1: Quantifying inter-pool and inter-individual variation in predatory behaviour. a) For each river pool, the apparatus used in the study was deployed in one of two treatments: in the prey treatment, 10 female guppies were placed within the cylinder (diagram not to scale), or in

112 an otherwise identical control apparatus without guppies (not shown). b) Depending on the level

113 of the analysis (inter-pool or inter-individual differences), the time spent near the stimulus was

114 defined as the total amount of time in which any pike cichlid (or a specific individual) was

115 present within a zone surrounding the stimulus during the 30 -minute presentation period. The green dashed line (approximately $24 \mathrm{~cm}$ from the outer edge of the cylinder) represents the outer

117 limit of the zone surrounding the stimulus, viewed from above. The total time spent near the

118 stimulus in each presentation is the sum of the time difference between $t_{1}$ and $t_{2}$ plus the time 119 spent within the zone during subsequent approaches (denoted by $t_{3}$ and $t_{4}$, etc.). c) Individual 
120 pike cichlids were identified through differences in humeral spot patterns (highlighted by green

121 circles), using still images recorded by the side-view GoPro video cameras.

123 Methods

124 Study site

125 We used a series of discrete natural river pools in the Lopinot valley, Trinidad (Appendix: Table

126 A1). Pools in this river are characterised by deeper areas of water $(>0.5 \mathrm{~m}$ in depth), bounded by

127 shallower sections containing riffles, rocks, boulders and small waterfalls which restrict the

128 movements of fish. Other, although minor, predators known to be present in this river include

129 blue acara cichlids (Aequidens pulcher), two-spot astyanax (Astyanax bimaculatus) and wolf-fish

130 (Hoplias malabaricus) (Magurran 2005).

Apparatus

The apparatus consisted of a transparent acrylic plastic cylinder (diameter: $15 \mathrm{~cm}$, height: $20 \mathrm{~cm}$,

134 wall thickness: $3 \mathrm{~mm}$ ) attached to a square base and covered by a perforated lid. Approaches to

135 the apparatus were recorded using Go Pro video cameras attached to the ends of three clear

136 acrylic plastic rods (diameter: $3 \mathrm{~cm}$, length: $80 \mathrm{~cm}$ ) secured to the base of the apparatus, with one

137 rod extending vertically and two identical rods projecting horizontally at right angles to one

138 another (Fig. 1a). This arrangement allowed footage of approaching fish to be captured from

139 above (Hero 3+ video camera, frame rate: 25 frames per second, resolution: 960p) and from the

140 side (two GoPro Hero 5 video cameras, frame rate: 30 frames per second, resolution: 2.7k;

141 diagonal field of view for both camera models: $133.6^{\circ}$ ). The apparatus was manoeuvred into 
142 position from the edge of each pool with minimal disturbance using transparent monofilament 143 attached to the base.

147 predators to their prey by repeatedly presenting a stimulus prey shoal (the prey treatment, 148 consisting of 10 female guppies placed within the cylinder of the apparatus) to free-swimming predatory fish in their natural environment. Control presentations of an otherwise identical apparatus without guppies were also conducted in the same locations to measure variation in the individual traits of the same predator individuals (such as personality variation in boldness and neophobia) which have been shown to influence encounter rates with prey. With this approach, it was then possible to test for the existence of a separate predator personality trait by examining individual responses to prey and responses of the same individuals when prey were absent. each 30-minute presentation period, the apparatus was placed in the same location and

157 orientation within each pool. For each river pool, the apparatus used in the study was deployed in 158 one of the two treatments in six separate presentations (once per day): three prey treatment 159 presentations and three control presentations. Presentations took place between 0730 and 1130 over a period of six consecutive days. Within each six-day period, control presentations of the 161 entirely novel apparatus always took place on the first day. Guppies used in prey treatment 162 presentations were caught from a single pool in the same river using a seine net (the stimulus 163 was not deployed in this pool). In each prey treatment presentation, 10 female guppies of a 
164

165

166

167

168

169

170

171

172

173

174

175

176

177

178

179

180

181

182

183

184

185

similar size were selected haphazardly from a number collected at the start of each day. The order in which the two treatments were assigned to the remaining five presentations per pool was randomised, but for logistical reasons, the three pools tested over the same six-day period shared the same presentation order. As incident light levels may affect pike cichlids' ability to detect prey, pool canopy openness was also measured after completion of the experiment by averaging measurements taken with a spherical densiometer in all four cardinal directions at three points along at the pool's upstream-downstream axis: the upstream end, midpoint and downstream end.

\section{Video analysis}

Data on the behavioural response of pike cichlids in each pool were extracted at two levels: poollevel data on the time spent near the stimulus by any predator individual over the course of the 30-minute presentation period, and individual-level data on the time spent near the stimulus by individual pike cichlids. In both the pool- and individual-level analyses, the time spent near the stimulus was defined as the total amount of time in which any pike cichlid (or a specific individual) was present within a zone surrounding the stimulus during the 30 -minute presentation period (the zone extended to approximately $24 \mathrm{~cm}$ from the outer edge of the cylinder; Fig. $1 \mathrm{~b}$ ). The number of pike cichlids in each pool was estimated using still images recorded using the side-view cameras (fish which could not be conclusively identified were not included in this total). The standard body length of individual pike cichlids (median approximate standard body length: $9.8 \mathrm{~cm}$, inter-quartile range: $2.9 \mathrm{~cm}$ ) was quantified in ImageJ (version 1.46r) using still images obtained from the downward-facing video camera (Fig. 1c) and comparing fish-length measurements in pixels to an object of known length (the stimulus cylinder viewed from above). 
All analyses were carried out using R v. 3.3.2 (R Development Core Team 2019), and all LMMs with the lme4 package. Results and further details for all models (including response variables, explanatory variables included as fixed effects, random effects and sample sizes for each model) are given in Appendix: Table A2. Prior to the analysis, all continous explanatory variables were standardised by subtracting the mean and dividing by the standard deviation. In each model, $P$ values for fixed effects were derived from likelihood ratio tests comparing the full model with a 195 reduced model lacking the variable in question. Model assumptions were verified by plotting residuals versus fitted values, using the DHARMa R package for GLMMs (Hartig 2019).

197 To quantify inter-individual differences in predatory behaviour, estimates of adjusted repeatabilities and inter-individual variances were obtained using the rptR package (Stoffel et al. 199 2017), which utilises a mixed model framework (Nakagawa and Schielzeth 2010), allowing experimental (presentation number and time of day) and environmental variables (canopy openness and the estimated number of pike cichlids in each pool) to be included as fixed effects.

202 Adjusted repeatabilities can thus be interpreted as a standardised measure of inter-pool or inter203 individual variation after potentially confounding variables have been controlled for. LMMs 204 were used to analyse pool-level data and Poisson GLMMs were used to analyse individual-level 205 data, which also included an approximate measure of pike cichlids' standard body length as an 206 additional fixed effect to control for predator body size. Pool identity was included as a random 207 intercept in models used to analyse pool-level data. Models used to analyse individual-level data 208 included both pool and individual identity as nested random intercepts. Statistical significance of 
209

210

211

212

213

214

215

216

217

218

219

220

221

222

223

224

225

226

227

228

229

230

231

repeatability estimates was assessed using both $P$-values (obtained through likelihood ratio tests) and overlap of the $95 \%$ confidence intervals with zero (computed via parametric bootstrapping).

Data on the time spent near the stimulus was censored at a maximum of 30 minutes

corresponding to the duration of a presentation, but no fish spent the maximum amount of time near the stimulus. Unless otherwise stated, instances when fish spent zero time near the stimulus were disregarded in the analysis in order to avoid influencing estimates of within-pool or withinindividual variation and thus affecting repeatabilities (Stamps et al. 2012).

Social interactions between predators within the same pool could potentially generate feedbacks which magnify (via differentiation) or suppress (via conformity) inter-individual differences in behaviour. To test for these possibilities, we conducted two types of randomisation simulations based on data for predators which approached the prey treatment stimulus in multiple presentations (44 individuals), following the methodology outlined in Ioannou et al. (2017). In the first randomisation, the mean observed pool-level diversity in the response to prey between predators across all pools was compared to the distribution of this statistic produced when the set of observations corresponding to an individual predator was randomly exchanged between pools. The aim of this approach was to determine whether the response of individual predators to prey was dependent on the other individuals present within the same pool, or whether being in a particular pool is statistically unimportant. Pool-level diversity in the response to prey was quantified as the coefficient of variation $(\mathrm{COV})$ in the time spent near the prey treatment stimulus. The second randomisation examined the relationship between the time spent near the stimulus by two individuals ('predator 1' and 'predator 2') which were randomly selected (without replacement) from the same pool. To enable direct comparison of the response to prey by 'predator 1' and 'predator 2', observations were selected from the same presentation per pair. 
232 Across multiple pools, a negative slope would be expected from behavioural differentiation

233 between the two individuals (if one predator spends a long time near the stimulus, the other does

234 not), whereas a positive slope would be consistent with conformity (both 'predator 1' and

235 'predator 2' spend a similar amount of time near the stimulus). The slope of the relationship

236 between time spent near the stimulus by 'predator 1' and 'predator 2' was estimated using a

237 quasi-Poisson generalised linear model, controlling for variation between pairs in presentation

238 number, time of day, canopy cover and the estimated number of pike cichlids in each pool by

239 including these variables as main effects. Both randomisation procedures were run with 10,000

240 iterations.

242 Ethical note

243 Ethical approval for all procedures was obtained through the University of Bristol (project

244 number UIN UB/17/006). The approach used in this study allowed the responses of free-

245 swimming pike cichlid predators to prey to be measured, whilst also protecting prey from harm,

246 through the presence of a physical barrier separating predators from prey. Guppies were

247 collected from a population in the Lopinot valley which naturally coexists with pike cichlids, and

248 after being used in the study, were returned to a pool in the same river.

$250 \quad \underline{\text { Results }}$

251 Responses to the experimental apparatus

252 A total of 69 individual pike cichlids were observed approaching the stimulus during at least one 253 30-minute presentation period (Appendix: Table A1). Compared to their behaviour in control 
presentations, pike cichlids spent more time near the stimulus when prey were present (Poisson generalised linear mixed effects model $(\mathrm{GLMM}), N_{\text {obs }}$ (no. of observations) $=211, N_{\text {ind }}$ (no. of individuals $)=69: \chi_{1}^{2}=63.2, P<0.001$; Appendix: Fig. A1 and Table A2, model 1). In addition, attacks on the stimulus, defined as a fast, directed movement towards the apparatus (Appendix: Video A1) were only observed in the prey treatment. Pike cichlids which spent more time near the stimulus were more likely to attack the stimulus during the same presentation (binomial GLMM, $N_{\text {obs }}=133, N_{\text {ind }}=68: \chi_{1}^{2}=41.1, P<0.001$; Fig. 2 ; Appendix: Table A2, model 3), and made more attacks during the first 30 seconds that they spent near the stimulus (Poisson GLMM, $N_{\text {obs }}=123, N_{\text {ind }}=63: \chi_{1}^{2}=6.83, P=0.009$; Fig. 2 ; Appendix: Table A2, model 5).

a)

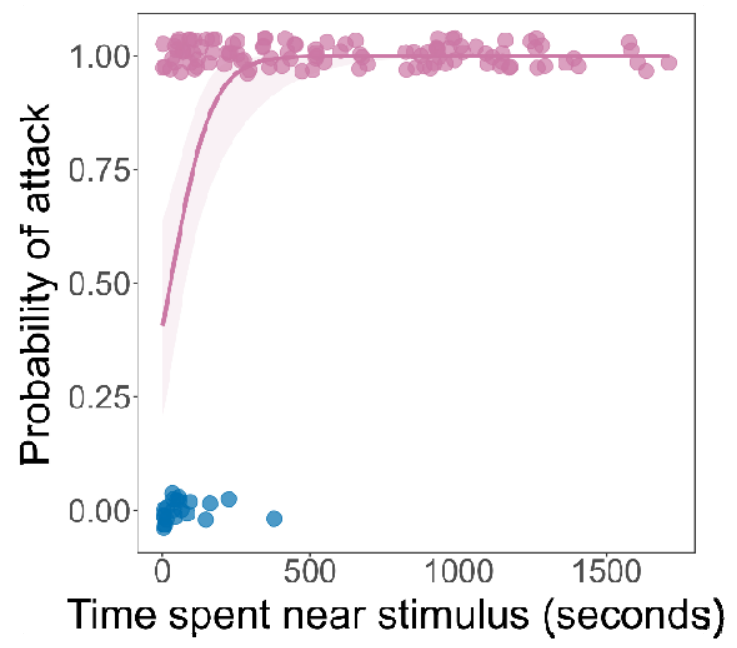

b)

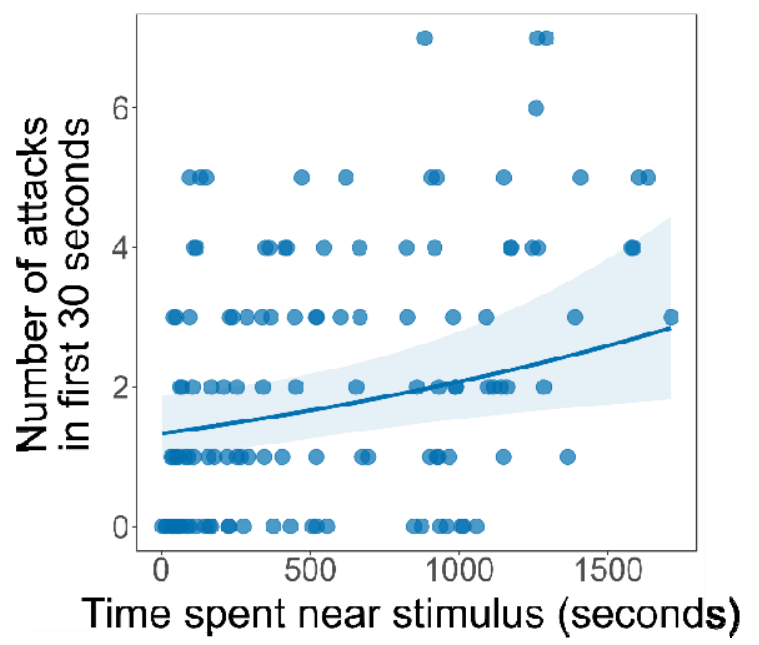
raw data to allow overlapping points to be visualised. b) Relationship between the number of attacks made by pike cichlids during the first 30 seconds they spent near the stimulus and the total time spent near the stimulus during each presentation (this analysis was limited to pike 
269 cichlids which spent a minimum of 30 seconds near the stimulus). In both a) and b), curves

270 represent the predicted response from a generalised linear mixed-effects model (GLMM): a

271 binomial GLMM featuring attacks on the stimulus as a binary response variable in a) and a

272 Poisson GLMM in b) (see Appendix: Table A2, models 3 and 5 respectively). Model predictions

273 were obtained by holding all other fixed effects constant at their mean values. Shading indicates

$27495 \%$ confidence intervals surrounding the predicted response.

275

276

Local variation in predation risk between river pools

277 From the prey's perspective, the repeatability of predation risk in their local habitat (pools in our

278 study) will be more relevant to the risk they experience than repeatable differences between

279 individual predators. We therefore quantified inter-pool variation in the time spent near the

280 stimulus by any pike cichlid in a given pool by estimating adjusted repeatabilities (Nakagawa

281 and Schielzeth 2010), controlling for variation arising from the experimental design (time of day

282 and presentation number) and environmental differences between the pools (the estimated

283 number of pike cichlids in each pool and canopy openness). Significant repeatability at the level

284 of the pool was evident during prey treatment presentations, even when controlling for the

285 estimated number of pike cichlids, i.e. predator density, in each pool $\left(R_{\text {pool }}=0.476,95 \%\right.$

286 confidence intervals: $0.093-0.759, P=0.009, N_{\text {obs }}=45, N_{\text {pool }}$ (no. of pools) $=16$; Fig. 3 ;

287 Appendix: Table A3). The time spent near the stimulus by pike cichlids was not repeatable

288 during control presentations without prey $\left(R_{\text {pool }}=0,95 \%\right.$ confidence intervals: $0-0.417, P=1$,

$289 \quad N_{\text {obs }}=35, N_{\text {pool }}=15$; Fig. 3; Appendix: Table A3). 


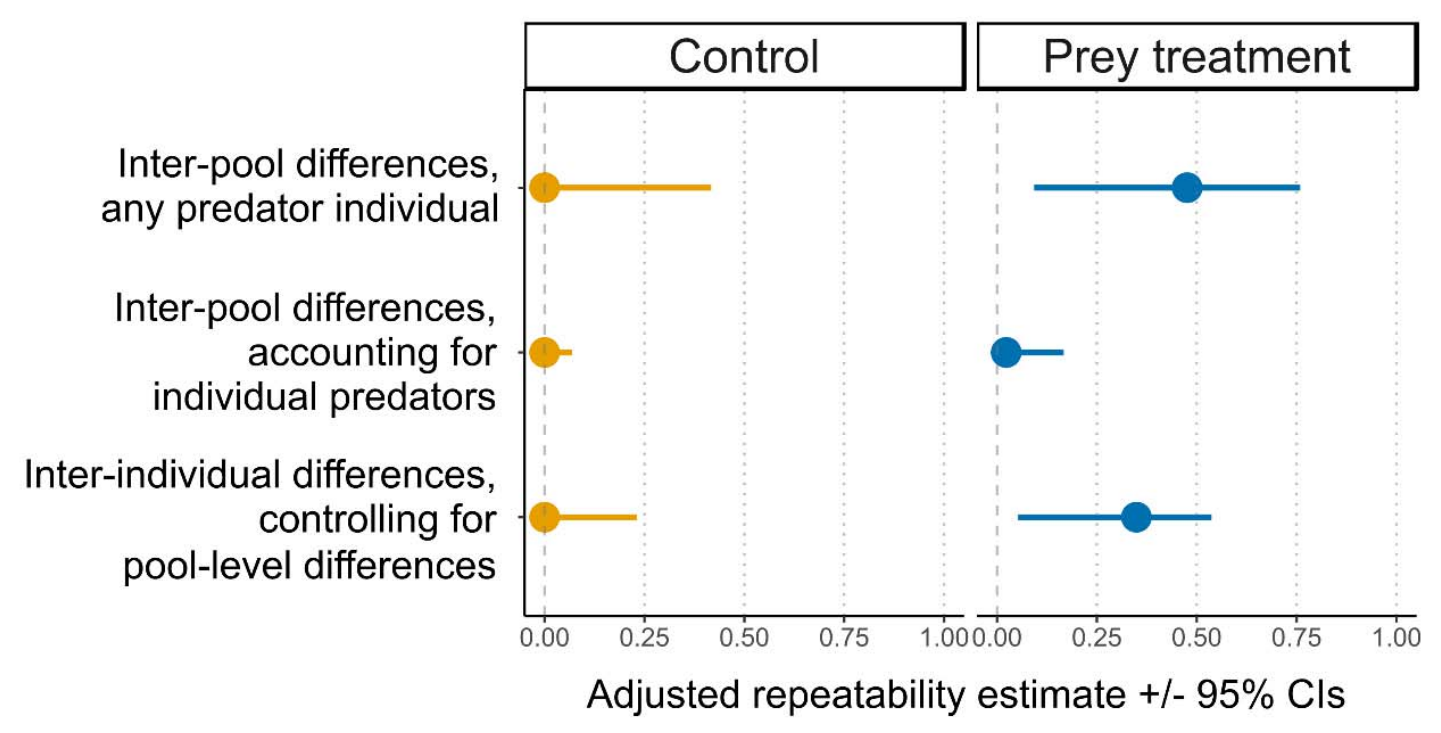

291 Figure 3: Pool- and individual-level repeatability estimates for the time spent near the stimulus,

292 during control (orange) and prey treatment (blue) presentations. The figure shows adjusted

293 repeatability estimates (filled circles), and the degree of uncertainty associated with each

294 estimate (error bars representing 95\% confidence intervals). Adjusted repeatabilities represent

295 the proportion of the total variance in the time spent near the stimulus which can be attributed to

296 variation between pools (top and middle rows) or individuals (bottom row), as opposed to

297 variation within pools or individuals. Whereas estimates of inter-pool differences in the upper

298 row are focused on the time spent near the stimulus by any predator individual (pool-level data,

299 Appendix: Table A3), those in the middle and lower rows are based on analysis of the time

300 individual predators spent near the stimulus (Appendix: Table A4).

302 Variation between individual predators in their response to prey 
To investigate inter-individual variation in predatory behaviour, adjusted repeatabilities were estimated for the time spent near the stimulus by individually-identified pike cichlids. This analysis was limited to pike cichlids which approached the stimulus in at least two separate prey treatment presentations, or in at least two separate control presentations. During prey treatment presentations, the time spent near the stimulus was significantly repeatable, even when controlling for standard body length and other experimental and environmental variables $\left(R_{\text {ind }}=\right.$ $0.349,95 \%$ confidence interval: $0.053-0.537, P=0.006, N_{\text {obs }}=109, N_{\text {ind }}=44$; Fig. 3 ; Appendix: Table A4). In contrast, repeatable inter-individual differences were not observed in the control treatment $\left(R_{\text {ind }}=0,95 \%\right.$ confidence interval: $0-0.231, P=0.5, N_{\text {obs }}=59, N_{\text {ind }}=24$; Fig. 3; Appendix: Table A4). Whilst the time spent near the stimulus was positively correlated across prey treatment presentations, no correlations were evident across control presentations (Appendix: Fig. A2). Additionally, with the identity of individual predators factored into these analyses, there was no longer any substantial inter-pool variation in the time spent near the stimulus $\left(R_{\text {pool }}=0.023,95 \%\right.$ confidence interval: $0-0.167, P=0.448, N_{\text {obs }}=109, N_{\text {pool }}=15$; Appendix: Table A4). This suggests that the inter-pool differences resulted from variation in the behaviour of individual predators in separate pools, rather than other sources of inter-pool variation such as predator density or environmental factors.

Possible effects of sampling bias and social interactions

Attempts to measure behaviour in natural populations are susceptible to bias because individuals vary in their tendency to engage with novel stimuli (Stuber et al. 2013), and because behavioural differences between individuals can be influenced by social interactions (McDonald et al. 2016). In our study, there was a positive association between the number of prey treatment presentations 
326 in which an individual approached the stimulus and the time it spent near the stimulus during the

327 first prey presentation it was observed in (Poisson GLMM, $N_{\text {obs }}=N_{\text {ind }}=68: \chi^{2}{ }_{1}=10.983, P<$

328 0.001; Appendix: Fig. A3 and Table A2, model 7). This implies that pike cichlids that

329 approached the stimulus in multiple presentations were more predatory on average, relative to

330 the overall predator population. The individual-level repeatabilities presented here (Fig. 3) may

331 therefore under-estimate the true extent of inter-individual variation as they are likely to exclude

332 the least predatory individuals. Randomisation simulations based on an approach developed by

333 Ioannou et al. (2017) suggested that diversity in the response to prey was not dependent on the

334 social environment within each pool (Fig. 4a). Additionally, there was no evidence for positive

335 or negative interactions between predators within the same pool (Fig. 4b), suggesting that the

336 observed inter-individual differences in predators' response to prey were not magnified or

337 suppressed as a result of social interactions.

a)

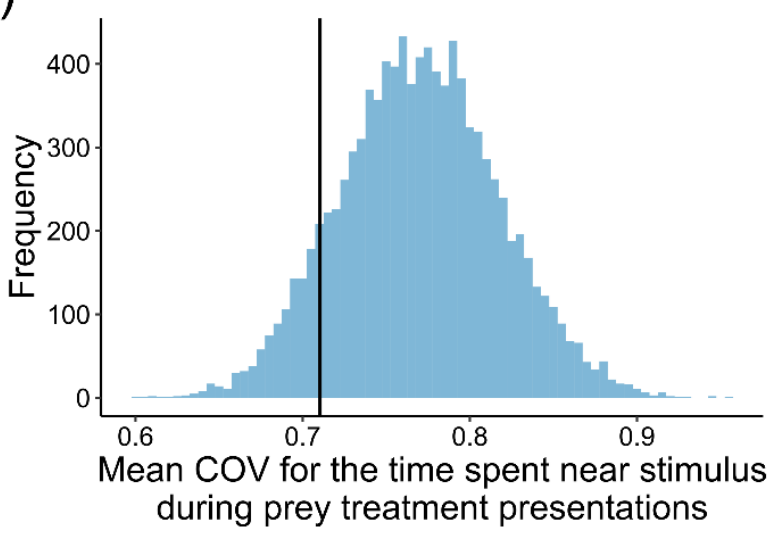

b)

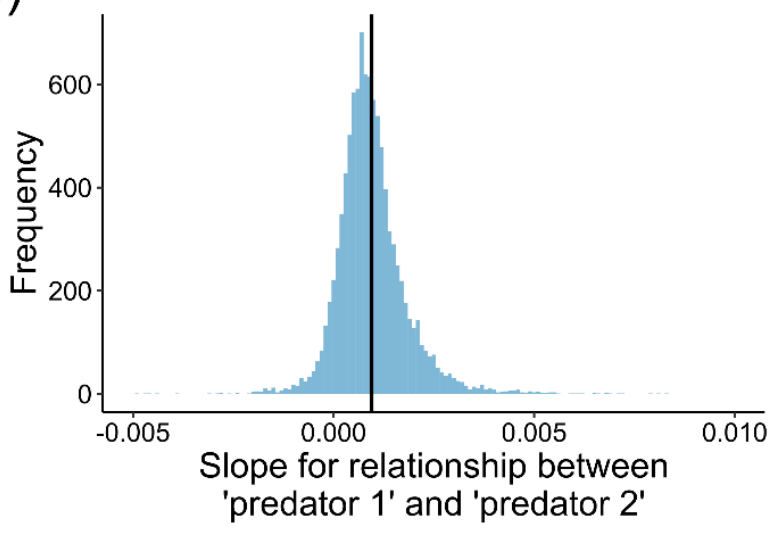

Figure 4: Results from randomisation simulations designed to examine the influence of social

341 interactions on inter-individual variation in predators' response to prey, based on 44 individual

342 predators which approached the prey treatment stimulus in multiple presentations. a) The 
343 observed mean group-level diversity $(\mathrm{COV})$ in predators' responses to prey (indicated by the

344 black vertical line) occurred within the $95 \%$ confidence intervals of the randomised distribution

345 for the same statistic, produced when observations corresponding to individual predators were

346 randomly shuffled between pools. b) The mean slope for the relationship between the time spent

347 near the stimulus by pairs of fish randomly selected from the same pool was weakly positive

348 (mean slope: 0.000976, shown by the black vertical line), but the $95 \%$ confidence intervals (-

$3490.000431,0.00299)$ of the randomised distribution of the slope overlapped with zero.

352 Predicting individual predatory responses from behaviour when prey are absent

353 The comparison of treatments with and without prey shows that the behaviour of individual

354 predators is only repeatable when prey are present. This suggests that the response to prey cannot

355 be explained by personality variation in boldness or neophobia, as consistent differences arising

356 from these traits should also lead to repeatability in the control presentations without prey. To

357 test this explicitly, we explored whether the behaviour of pike cichlids during control treatment

358 presentations could account for the repeatability in their response during prey treatment

359 presentations. Adjusted repeatability estimates in the time spent near the stimulus during the prey

360 treatment remained significantly repeatable when the mean time spent near the stimulus during

361 control presentations was included as a covariate $\left(N_{\text {obs }}=109, N_{\text {ind }}=44: R_{\text {ind }}=0.33,95 \%\right.$

362 confidence interval: $0.013-0.506, P=0.003)$. The repeatability estimate was still statistically

363 significant when the time spent near the stimulus during the first control presentation an

364 individual was observed in was instead included as a covariate $\left(N_{\text {obs }}=109, N_{\text {ind }}=44: R_{\text {ind }}=\right.$ 
0.294, $95 \%$ confidence interval: $0.021-0.508, P=0.006$; in both analyses, individuals were assigned zero time spent near the stimulus in the control if they were not observed in the control treatments). These results are consistent with the absence of any correlations between the time spent near the stimulus during prey treatment presentations and the mean time spent near the stimulus across control presentations or time spent near the stimulus during the first control presentation in which an individual was observed (Appendix: Fig. A4 and Table A2: models 89). There was however a near-significant tendency for individuals observed in control presentations to spend more time near the prey treatment stimulus than those individuals which were never observed in the control (Appendix: Fig. A5 and Table A2, models 10-11), providing some indication of a role of boldness in determining the response of individual predators to their prey.

\section{Components of repeatability in predatory behaviour}

One explanation for the increased repeatability of behaviour in the prey treatment compared to the control is that variation between individuals is greater in the presence of prey. To examine this possibility, we estimated inter-individual variances for the time spent near the stimulus during the control and prey treatments, which, unlike estimates of the repeatability, are not influenced by the consistency of individual behaviour (i.e. intra-individual variation) within each treatment. In contrast to negligible inter-individual variability among fish observed across control presentations $\left(N_{\text {obs }}=59, N_{\text {ind }}=24\right.$, inter-individual variance: $0,95 \%$ confidence interval: $0-0.353)$, inter-individual variability was apparent in the prey treatment $\left(N_{\text {obs }}=109, N_{\text {ind }}=44\right.$, inter-individual variance: $0.597,95 \%$ confidence interval: $0.064-0.977$; Fig. 5). The residual variance was also higher in the control (1.645, 95\% confidence interval: 0.844 - 2.118) compared 
to the prey treatment $(1.074,95 \%$ confidence interval: $0.713-1.49)$, suggesting that intraindividual variation was lower when prey were present. Thus, the significant repeatability between individuals in the prey treatment and the lack of repeatability in the control can be explained by individuals being more variable relative to one another and also behaving more consistently in the prey treatment.

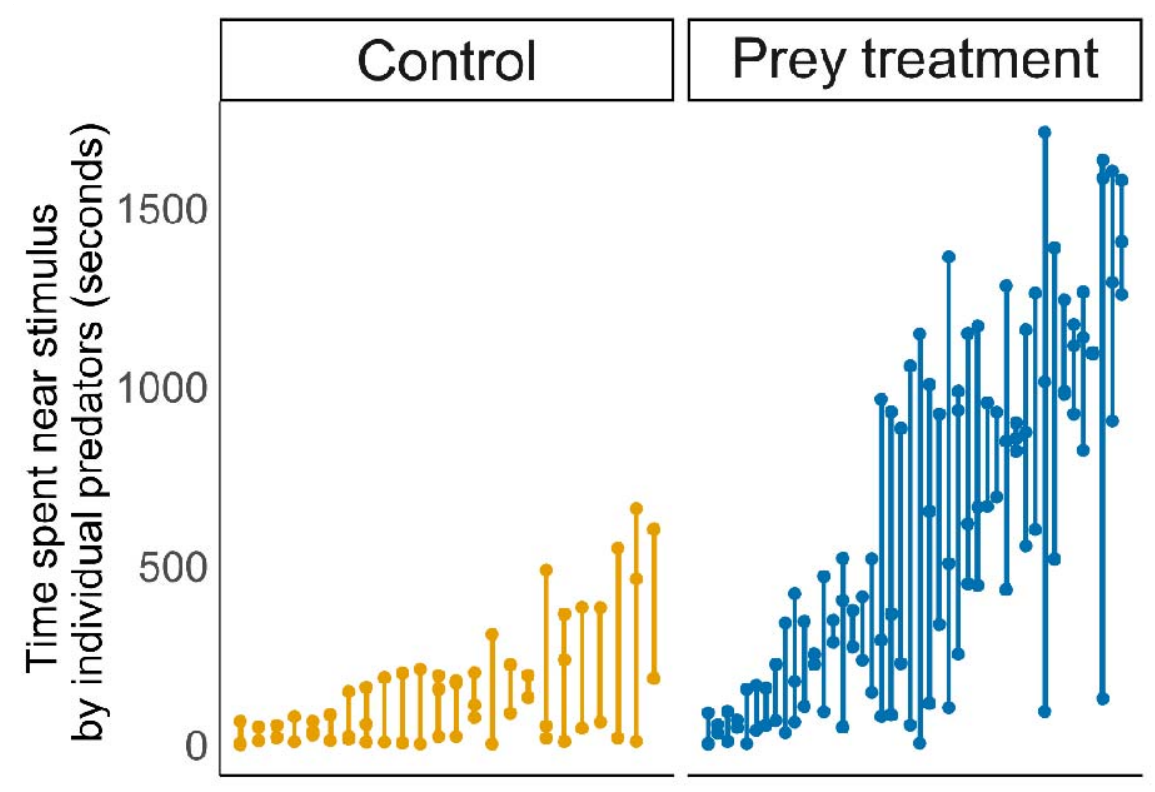

393

394 Figure 5: Variability in the behaviour of individual predators in the control (orange) and prey

395 treatments (blue). Points indicate the time spent near the stimulus in each presentation, and the

396 vertical lines span the range for each individual. Data is shown for individuals which approached

397 the stimulus in two or more prey treatment presentations $\left(N_{\text {ind }}=44\right)$ or two or more control

398 presentations $\left(N_{\text {ind }}=24\right)$, ordered by increasing mean time spent near the stimulus. 
400

401

402

403

404

405

406

407

408

409

410

411

412 417 response to prey.

\section{$\underline{\text { Discussion }}$}

By studying the response of wild piscivorous fish to a stimulus prey shoal across a series of natural river pools, our study provides evidence for consistent inter-individual variation in the response of predators to their prey. After controlling for their body size and for experimental and environmental factors, we found that individual pike cichlids differed consistently in the time spent near the stimulus when prey were present, but not when prey were absent. Crucially, our analysis revealed that inter-individual variation in predatory behaviour could not be explained by the response of predators to the empty presentation apparatus (a novel object). This indicates that the response to prey reflects a personality trait which is specific to predation, is distinct from other commonly measured personality traits such as boldness or neophobia, and is independent of the previously documented correlation between boldness and encounter rates with prey (Pruitt et al. 2012). This individual-level trait accounted for variation between pools in the time spent near the stimulus by any pike cichlid, suggesting it is likely to explain a significant proportion of the risk faced by prey in their local environment, even when accounting for predator density. Our analysis also shows that inter-individual differences in the response to prey were unlikely to have arisen from pre-existing variation between predators becoming magnified due to the presence of prey, as boldness (as measured during control presentations) did not predict individual predators'

The existence of a personality trait specific to predation could affect prey in two main ways: by influencing the strength of non-lethal effects on prey traits, or by directly affecting survival during predator-prey encounters. Most obviously, the sustained or repeated presence of a nearby predator is likely to affect the level of risk perceived by prey. Prey often respond to the

422 heightened risk of predation with a change in their behaviour, for example by increasing their 
423 vigilance, shifting microhabitat use or by forming social groups (Lima and Dill 1990). While

424 anti-predator responses can be effective in enhancing survival over the short-term, these

425 responses often reduce the amount of time allocated to foraging or reproductive behaviour,

426 leading to long-term fitness costs. In many instances, the potency of non-lethal predator effects

427 on prey fitness components such as individual growth rates, survival or reproduction can rival

428 the impact of direct prey consumption (Preisser et al. 2005), with potentially substantial

429 consequences for prey demographic rates (Eggers et al. 2006), prey population dynamics

430 (Peckarsky et al. 2008), interactions between prey and their competitors (Werner 1991) and the

431 abundance of resources at lower trophic levels (Suraci et al. 2016). The behavioural response of

432 prey to predation risk will depend on their perception of the impending threat, which will be

433 sensitive to how predators in the vicinity are behaving (Stankowich and Blumstein 2005). For

434 prey, a predator personality trait should make the level of background predation risk perceived

435 by prey more predictable, particularly in situations where the same predator and prey individuals

436 encounter one another frequently, as is the case in relatively isolated pools within the guppy-pike

437 cichlid system.

438 A predation-specific personality trait could have a strong influence on the eventual outcome of

439 encounters with prey and help to maintain behavioural variation within the prey population

440 (McGhee et al. 2013). While our study was not designed to explore the possible direct effects of

441 predator personality on prey survival, our results suggest that inter-individual variation in the

442 predatory behaviour measured here may have potentially lethal consequences for prey. Although

443 we found a positive association between the time spent near the stimulus and the overall

444 probability of attack by pike cichlids, this association might be expected if the number of

445 predatory strikes simply grows linearly with time. However, the positive relationship between 
446 the time spent by predators near the stimulus and the initial rate of attack (during the first 30

447 seconds spent near the stimulus) also implies that predators which spend more time around prey

448 are more motivated to attack when prey are first encountered, and thus pose a greater threat.

449 Having established that individual predators consistently differ in their response to a standardised 450 prey stimulus independently of boldness, future research could investigate the impact of these

451 differences on dynamic interactions between predators and prey, in a setting where prey are

452 unconstrained and both predators and prey are free to respond to cues from one another. This

453 would address whether prey adjust their anti-predator behaviour in response to predation-specific

454 personality variation. If carried out in a setting where prey were exposed to individual predators

455 for an extended period, this could also help clarify the relative impact on prey survival of a

456 predation-specific personality trait compared to variation between individual predators in

457 commonly studied personality traits which affect encounter rates, such as boldness or activity.

458 We also found evidence that consistent inter-individual variation in the behaviour of the predator

459 individuals present within each pool accounted for differences between pools in the time spent

460 near the stimulus by any pike cichlid. Pool-level differences were not correlated with the

461 densities of predators in each pool, suggesting that the personality traits of resident predators

462 could be an important factor contributing to local differences in predation risk. However, in

463 addition to predator density, pools might also differ in the social environment predators are

464 exposed to. If the presence of an individual near the stimulus alerts others to the presence of prey

465 through social information (Pitcher et al. 1982), inter-individual differences might be suppressed

466 by social interactions. Alternatively, if socially dominant predators aggressively exclude

467 subordinates from accessing prey, feedbacks resulting from differences in social dominance

468 could also magnify inter-individual variation in the response to prey (Bergmüller and Taborsky 
2010). It was therefore important to account for the possibility that variation between pools in the

470

471

472

473

474

475

476

477

478

479

480

481

482

483

484

485

486

487

488

489

490

491 nature and strength of social interactions could generate the observed inter-individual differences in predator behaviour (McDonald et al. 2016). In our study, randomisation simulations showed that the degree of inter-individual variation in the time spent near prey was not dependent on the observed distribution of individual pike cichlids between pools. This suggests that there were no social interactions between individual predators that positively or negatively affected interindividual variation. In other words, being present within a particular pool, and exposed to a particular set of other individuals with specific behavioural characteristics, did not have a strong influence on an individual predator's response to prey. Although it is difficult to fully disentangle intrinsic inter-individual differences from environmental influences on behaviour without translocating animals and quantifying their behaviour in different contexts (Niemelä and Dingemanse 2017), these findings support the conclusion that stable differences in social interactions were unlikely to have played a major role in shaping the observed inter-individual variation in the response to prey.

By demonstrating that the risk prey experience cannot always be adequately predicted from frequently studied axes of personality variation, our study highlights the importance of considering inter-individual variation in traits with direct ecological relevance. These results also have specific implications for the guppy-pike cichlid system, in which geographically isolated guppy populations occupying low- and high-predation environments demonstrate dramatic differences in numerous aspects of their life history (Reznick et al. 1990) and behaviour (Ioannou et al. 2017). Since high-predation zones are characterised by the presence of pike cichlids, the existence of differences in predator behaviour between pools adds to the accumulating evidence that predation pressure is more heterogeneous within these areas than 
492 implied by the well-studied contrast between high- and low-predation environments (Deacon et

493 al. 2018).

$\underline{\text { Acknowledgments }}$

496 We thank Robert Heathcote and Darren Croft for advice in the field, and thank Emmanuelle

497 Briolat, Hannah MacGregor, Iestyn Penry-Williams and Teresa Szopa for helpful comments on

498 the manuscript. This work was supported by a NERC GW4+ Doctoral Training Partnership

499 studentship from the Natural Environment Research Council [NE/L002434/1] awarded to ASC,

500 and a Natural Environment Research Council Fellowship [NE/K009370/1] and a Leverhulme

501 Trust grant [RPG-2017-041 V] awarded to CCI.

504 Ballew, N. G., G. G. Mittelbach, and K. T. Scribner. 2017. Fitness Consequences of Boldness in 505 Juvenile and Adult Largemouth Bass. American Naturalist 189: 396-406.

506 Beckerman, A. P., M. Uriarte, and O. J. Schmitz. 1997. Experimental evidence for a behavior507 mediated trophic cascade in a terrestrial food chain. Proceedings of the National Academy of 508 Sciences USA 94: 10735-10738.

509 Bell, A. M., and A. Sih. 2007. Exposure to predation generates personality in threespined 510 sticklebacks (Gasterosteus aculeatus). Ecology Letters 10: 828-834.

511 Bell, A. M., S. J. Hankison, and K. L. Laskowski. 2009. The repeatability of behaviour: a meta512 analysis. Animal Behaviour 77: 771-783. 
513 Bergmüller, R., and M. Taborsky. 2010. Animal personality due to social niche specialisation.

514 Trends in Ecology and Evolution 25: 504-511.

515 Botham, M. S., C. J. Kerfoot, V. Louca, and J. Krause. 2006. The effects of different predator 516 species on antipredator behaviour in the Trinidadian guppy, Poecilia reticulata.

$517 \quad$ Naturwissenschaften 93: 431-439.

518 Chang, C., H. Y. Teo, Y. Norma-Rashid, and D. Li. 2017. Predator personality and prey

519 behavioural predictability jointly determine foraging performance. Scientific Reports 7: 40734.

520 Cote, J., S. Fogarty, K. Weinersmith, T. Brodin, and A. Sih. 2010. Personality traits and dispersal

521 tendency in the invasive mosquitofish (Gambusia affinis). Proceedings of the Royal Society B

$522 \quad 277: 1571-1579$.

523 Croft, D. P., L. J. Morrell, A. S. Wade, C. Piyapong, C. C. Ioannou, J. R. G. Dyer, B. B.

524 Chapman, Y. Wong, and J. Krause. 2006. Predation risk as a driving force for sexual

525 segregation: a cross-population comparison. American Naturalist 167: 867-78.

526 Dall, S. R. X., A. M. Bell, D. I. Bolnick, and F. L. W. Ratnieks. 2012. An evolutionary ecology

527 of individual differences. Ecology Letters 15: 1189-1198.

528 Deacon, A. E., F. A. M. Jones, and A. E. Magurran. 2018. Gradients in predation risk in a

529 tropical river system. Current Zoology 64: 213-221.

530 Des Roches, S., D. M. Post, N. E. Turley, J. K. Bailey, A. P. Hendry, M. T. Kinnison, J. A.

531 Schweitzer, and E. P. Palkovacs. 2018. The ecological importance of intraspecific variation.

$532 \quad$ Nature Ecology and Evolution 2: 57-64. 
Hartig, F. 2019. DHARMa: Residual Diagnostics for Hierarchical (Multi-Level / Mixed)

Regression Models. R package version 0.2.5.

Eggers, S., M. Griesser, M. Nystrand, and J. Ekman. 2006. Predation risk induces changes in nest-site selection and clutch size in the Siberian jay. Proceedings of the Royal Society B 273: 701-706.

Firth, J. A., E. F. Cole, C. C. Ioannou, J. L. Quinn, L. M. Aplin, A. Culina, K. McMahon, and B. C. Sheldon. 2018. Personality shapes pair bonding in a wild bird social system. Nature Ecology and Evolution 2: 1696-1699.

Heathcote, R. J. P., J. Troscianko, S. K. Darden, L. C. Naisbett-Jones, P. L. Laker, A. M. Brown, I. W. Ramnarine, J. Walker, and D. P. Croft. 2020. A matador-like predator diversion strategy driven by conspicuous colouration in guppies. Current Biology 30, 1-8.

Hulthén, K., B. B. Chapman, P. A. Nilsson, L. A. Hansson, C. Skov, J. Brodersen, J. Vinterstare, and C. Brönmark. 2017. A predation cost to bold fish in the wild. Scientific Reports 7: 1239.

Ioannou, C. C., M. Payne, and J. Krause. 2008. Ecological consequences of the bold-shy continuum: The effect of predator boldness on prey risk. Oecologia 157: 177-182.

Ioannou C. C., I. W. Ramnarine, and C. J. Torney. 2017. High-predation habitats affect the social dynamics of collective exploration in a shoaling fish. Science Advances 3: e1602682.

Koski, S. E. 2014. Broader horizons for animal personality research. Frontiers in Ecology and Evolution 2: 70.

Lima, S. L. 1998. Nonlethal Effects in the Ecology of Predator-Prey Interactions. Bioscience 48: 25-34. 
554 Lima, S. L. 2002. Putting predators back into behavioral predator-prey interactions. Trends in 555 Ecology and Evolution 17: 70-75.

556 Lima, S. L., and L. M. Dill. 1990. Behavioral decisions made under the risk of predation: a

557 review and prospectus. Canadian Journal of Zoology 68: 619-640.

558 MacGregor, H. E. A., J. E. Herbert-Read, and C. C. Ioannou. 2020. Information can explain the 559 dynamics of group order in animal collective behaviour. Nature Communications 11, 2737.

560 Magurran, A. E. 2005. Evolutionary ecology: the Trinidadian guppy. Oxford, UK: Oxford 561 University Press.

562 McDonald, N. D., S. A. Rands, F. Hill, C. Elder, and C. C. Ioannou. 2016. Consensus and 563 experience trump leadership, suppressing individual personality during social foraging. Science 564 Advances 2: e1600892.

565 McGhee, K. E., L. M. Pintor, and A. M. Bell. 2013. Reciprocal Behavioral Plasticity and 566 Behavioral Types during Predator-Prey Interactions. American Naturalist 182: 704-717.

567 Michalko R., and R. Řežucha. 2018 Top predator's aggressiveness and meso-predator's risk568 aversion additively determine probability of predation. Behavioral Ecology and Sociobiology $569 \quad 72: 105$

570 Moiron, M., K. L. Laskowski, and P. T. Niemelä. 2020. Individual differences in behaviour 571 explain variation in survival: a meta-analysis. Ecology Letters 23: 399-408.

572 Nakagawa, S., and H. Schielzeth. 2010. Repeatability for Gaussian and non-Gaussian data: A 573 practical guide for biologists. Biological Reviews 85: 935-956. 
574 Niemelä, P. T., and N. J. Dingemanse. 2014. Artificial environments and the study of 'adaptive'

575 personalities. Trends in Ecology and Evolution 29: 245-247.

576 Niemelä, P. T., and N. J. Dingemanse. 2017. Individual versus pseudo-repeatability in behaviour:

577 Lessons from translocation experiments in a wild insect. Journal of Animal Ecology 86: 1033-

5781043.

579 Okuyama, T. 2008. Individual behavioral variation in predator-prey models. Ecological

580 Research 23: 665-671.

581 Paine, R. T. 1966. Food Web Complexity and Species Diversity. America Naturalist 100: 65-75.

582 Patrick, S. C., D. Pinaud, and H. Weimerskirch. 2017. Boldness predicts an individual's position

583 along an exploration-exploitation foraging trade-off. Journal of Animal Ecology 86: 1257-1268.

584 Peckarsky, B. L., P. A. Abrams, D. I. Bolnick, L. M. Dill, J. H. Grabowski, B. Luttbeg, J. L.,

585 Orrock, S. D. Peacor, E. L. Preisser, O. J. Schmitz, and G. J. Trussell. 2008. Revisiting the

586 classics: considering nonconsumptive effects in textbook examples of predator-prey interactions.

587 Ecology 89: 2416-25.

588 Pitcher, T. J., A. E. Magurran, and I. J. Winfield. Fish in larger shoals find food faster.

589 Behavioral Ecology and Sociobiology 10: 149-151.

590 Preisser, E. L., D. I. Bolnick, and M. F. Benard. 2005. Scared to death? The effects of

591 intimidation and consumption in predator-prey interactions. Ecology 86: 501-509.

592 Pruitt, J. N., J. J. Stachowicz, and A. Sih. 2012. Behavioral Types of Predator and Prey Jointly

593 Determine Prey Survival: Potential Implications for the Maintenance of Within-Species

594 Behavioral Variation. American Naturalist 179: 217-227. 
595 R Development Core Team. 2019. R: A language and environment for statistical computing. R

596 Foundation for Statistical Computing, Vienna, Austria.

597 Réale, D., S. M. Reader, D. Sol, P. T. McDougall, and N. J. Dingemanse. 2007. Integrating

598 animal temperament within ecology and evolution. Biological Reviews 82: 291-318.

599 Reznick, D. A., H. Bryga, and J. A. Endler. 1990. Experimentally induced life-history evolution 600 in a natural population. Nature 346: 357-359.

601 Rhoades, O. K., S. I. Lonhart, and J. J. Stachowicz. 2019. Human-induced reductions in fish

602 predator boldness decrease their predation rates in kelp forests. Proceedings of the Royal Society

603 B 286: 20182745.

604 Schmitz, O. J., V. Krivan, and O. Ovadia. 2004. Trophic cascades: The primacy of trait-mediated 605 indirect interactions. Ecology Letters 7: 153-163.

606 Sih, A., J. Cote, M. Evans, S. Fogarty, and J. N. Pruitt. 2012. Ecological implications of

607 behavioural syndromes. Ecology Letters 15: 278-289.

608 Spiegel, O., S. T. Leu, A. Sih, S. G. Godfrey, and C. M. Bull. 2015. When the going gets tough:

609 behavioural-type dependent space use in the sleepy lizard changes as the season dries.

$610 \quad$ Proceedings of the Royal Society B 282: 20151768.

611 Smith, B. R., and D. T. Blumstein. 2008. Fitness consequences of personality: A meta-analysis.

612 Behavioral Ecology 19: 448-455.

613 Stamps, J. A., M. Briffa, and P. A. Biro. 2012. Unpredictable animals: Individual differences in 614 intraindividual variability (IIV). Animal Behavior 83: 1325-1334. 
615 Stankowich, T., and D. T. Blumstein. 2005. Fear in animals: A meta-analysis and review of risk

616 assessment. Proceedings of the Royal Society B 272: 2627-2634.

617 Start, D., and B. Gilbert. 2017. Predator personality structures prey communities and trophic 618 cascades. Ecology Letters 20: 366-374.

619 Szopa-Comley, A. W., W. G. Donald, and C. C. Ioannou. 2020. Predator personality and prey 620 detection: inter-individual variation in responses to cryptic and conspicuous prey. Behavioral 621 Ecology and Sociobiology 74: 70.

622 Stuber, E. F., Y. G. Araya-Ajoy, K. J. Mathot, A. Mutzel, M. Nicolaus, J. J. Wijmenga, J. C.

623 Mueller, and N. J. Dingemanse. 2013. Slow explorers take less risk: A problem of sampling bias 624 in ecological studies. Behavioral Ecology 24: 1092-1098.

625 Stoffel, M. A., S. Nakagawa, and H. Schielzeth. 2017. rptR: repeatability estimation and variance 626 decomposition by generalized linear mixed-effects models. Methods in Ecology and Evolution 8:

$627 \quad 1639-1644$.

628 Suraci, J. P., M. Clinchy, L. M. Dill, D. Roberts, and L. Y. Zanette. 2016. Fear of large

629 carnivores causes a trophic cascade. Nature Communications 7: 10698.

630 Toscano, B. J., and B. D. Griffen. 2014. Trait-mediated functional responses: Predator

631 behavioural type mediates prey consumption. Journal of Animal Ecology 83: 1469-1477.

632 Walker, J. A., C. K. Ghalambor, O. L. Griset, D. McKenney, and D. N. Reznick. 2005. Do faster 633 starts increase the probability of evading predators? Functional Ecology 19: 808-815.

634 Werner, E. E. 1991. Nonlethal Effects of a Predator on Competitive Interactions Between Two 635 Anuran Larvae. Ecology 72: 1709-1720. 